\title{
Dynamic control of the focal light distribution with pupil masks
}

\section{Vidal Canales, Pedro Valle, José Oti, Manuel Cagigal}

Vidal F. Canales, Pedro J. Valle, José E. Oti, Manuel P. Cagigal, "Dynamic control of the focal light distribution with pupil masks," Proc. SPIE 9664, Ninth International Topical Meeting on Education and Training in Optics and Photonics, 966427 (24 October 2005); doi: 10.1117/12.2207689

SPIE Event: Ninth International Topical Meeting on Education and Training in Optics and Photonics, 2005, Marseille, France 
This paper is freely available as a resource for the optics and photonics education community.

Ref ETOP031

Dynamic control of the focal light distribution with pupil masks

Vidal F. Canales, Pedro J. Valle, José E. Oti and Manuel P. Cagigal

Departamento de Física Aplicada. Universidad de Cantabria, Los Castros S/N, 39005. Santander, Spain, fernancv@unican.es

\begin{abstract}
The light intensity distribution near focus of an optical system can be modified by pupil-plane masks. In fact, in some relevant fields, such as optical data storage or confocal microscopy, there is an increasing interest in the design of pupil masks for controlling the Point Spread Function (PSF). These techniques present a great advantage for educational purposes, since they deal with current technology but at the same time illustrate basic diffraction principles. In this work, we introduce a simple low-cost set up that allows the dynamical control of the PSF and can be easily implemented in any educational centre.
\end{abstract}

\title{
Keywords \\ Superresolution, Image forming and processing.
}

\section{Summary}

The control of the light intensity distribution near the geometrical focus of an optical system is an interesting topic both in industrial applications and for educational purposes. The design of pupil masks for this task has been approached from quite different points of view. Some masks are based on variable transmittance pupils 1 or on hybrid amplitude-phase profiles 2 while other use phase-only profiles because these perform better than transmittance masks.3 Phase-only masks can be classified in continuous 4 and annular profiles, which are more frequently used because of their in comparison, best performance and simplicity. Furthermore, they are easy to manufacture for mass production using available diffractive optics production methods and replication technologies. Some examples of annular masks are the diffractive superresolution elements, 5 the three zones binary phase filters designed to increase the data storage density in DVDs6 or the $\mathrm{N}$-zones binary filters that provide transverse, axial or 3D superresolution.7 Moreover, the parameters of the latter masks can be analytically derived from the figures of merit that describe the light intensity distribution near the geometrical focus: the Strehl ratio, the transverse and axial gain factors and the focus displacement.7,8 The Strehl ratio $S$ is a relevant parameter for analyzing image quality and is defined as the ratio of the intensity at the focal point to that corresponding to an unobstructed pupil. The transverse gain GT gives a measure of the superresolution performance in the transverse direction. The same applies for the axial gain, GA. These factors are normalized so that for the unobstructed pupil they are equal to unity. In a particular direction, a filter is superresolving when the corresponding gain is greater than unity and it is an apodizer when the corresponding gain is lower than unity. These figures of merit are obtained in terms of the moments of the pupil function7,9 and, consequently, are related to the mask parameters. Thus, pupil masks with any specific behavior can be designed for many different fields as for example in optical data storage, confocal microscopy, astronomy, laser printing, free-space communications or 3D imaging. 
However, the use of pupil-plane masks for PSF control presents a limitation. Once a mask is fabricated the performance is fixed and if we desire to change any of the PSF characteristics (e.g. resolution) we need to change the mask. Nonetheless, we introduce a technique to overcome this drawback. It is based on the control of the transmittance of each of the mask zones by simple means such as opaque masks, diaphragms or linear polarizers. Figure 1 shoes a simple scheme for this technique that only requires non-expensive elements that are present in any laboratory. Note that the $0-\square$ mask can be realized by cutting an annulus in any standard plastic sheet that presents $\square$ phase for the monochromatic source used, and that the polarizers can be substituted by a diaphragm. This set up allows us to modify at will the PSF of a fixed pupil-plane mask. In fact, the figures of merit of the PSF present a continuous variation, as shown in figure 2. This technique for varying the PSF characteristics is useful for any kind of mask, although it is best suited for annular binary phase-only masks. By the way, an alternative solution is to implement masks in a dynamic device such as a SLM, which is more expensive, or to use a Radial Birrefringent Element, which is less flexible and offers lower performance than our scheme.

The technique for PSF control presented in this work presents a great advantage for photonics education: on one hand, it is useful in up to date technology but at the same time it illustrates basic diffraction principles and a non-expensive set up can be easily built in any education centre. This combination of deep theoretical basis and industrial innovation is essential in the evolution of our educational systems towards European Higher Education Area.

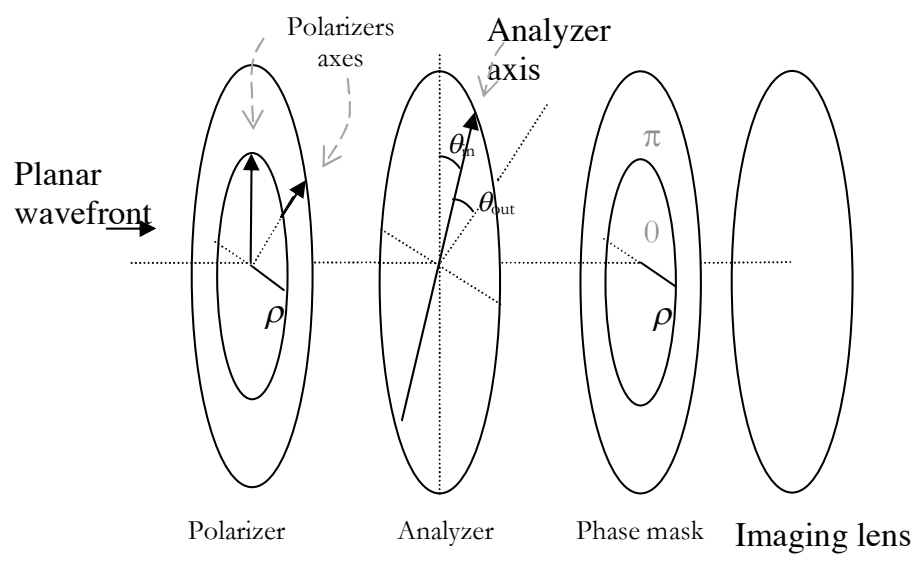

Figure 1. Scheme of the system that allows the control of the light intensity distribution with a fixed pupil-plane mask. It consists of a standard polarizer of the same size as the phase-only mask (analyzer) and another polarizer with two zones that can rotate with respect to each other: an inner circle and an outer annulus of the same size as those of the phase-only mask. 


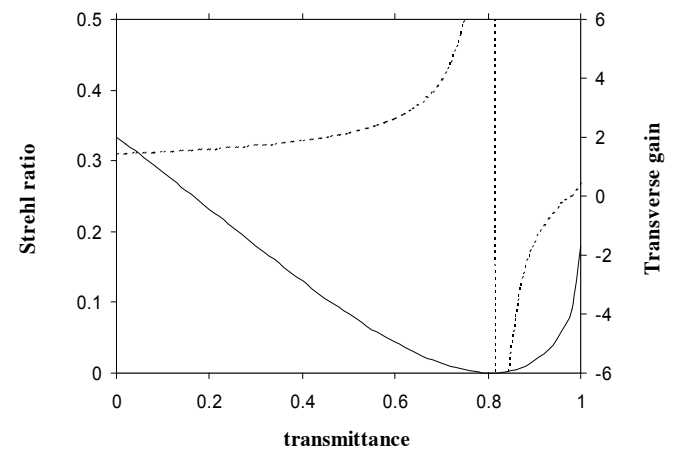

Figure 2. Strehl ratio (solid curve) and transverse gain (dashed curve) as a function of the transmittance of the inner zone for a mask of normalized radius $\square 1=0.65$, inner circle with phase 0 and outer annular zone of transmittance tout=(1- tin2)1/2 and phase $\square$.

\section{References}

[1] G. Toraldo di Francia, Nuovo Cimento Suppl. 9 (1952) 426-435.

[2] Z. S. Hegedus and V. Sarafis, J. Opt. Soc. Am. 3 (1986) 1892-1896.

[3] T. R. M. Sales and G. M. Morris, Opt. Lett. 22 (1997) 582-584.

[4] D.M. de Juana, J.E. Oti, V.F. Canales and M.P. Cagigal, Opt. Lett. 28 (2003) 607-609.

[5] T.R.M. Sales and G.M. Morris, J. Opt. Soc. Am. 14 (1997) 1637-1646.

[6] H. Wang, Z. Chen and F. Gan, Appl. Opt. 40 (2001) 5658-5662.

[7] V.F.,Canales J.E. Oti and M.P. Cagigal, Opt. Commun. 247 (2005) 11-18

[8] C.J.R. Sheppard and Z.S. Hegedus, J. Opt. Soc. Am. 5 (1988) 643-647.

[9] D.M. de Juana, J.E. Oti, V.F. Canales and M.P. Cagigal, JOSA A 20, (2003) 2172-2178 